\title{
STABILITY OF SOLUTIONS OF LINEAR SYSTEMS WITH DOMINANT MAIN DIAGONAL
}

\author{
CHARLES KAHANE
}

\begin{abstract}
We establish the asymptotic stability of solutions of a first order linear system of differential equations in which the matrix characterizing the system has a dominating principal diagonal with negative entries; the domination is expressed through the condition that, numerically, each entry in the principal diagonal exceeds the sum of the absolute values of the remaining entries in the same column.
\end{abstract}

The purpose of this note is to establish the following

THEOREM. All solutions of the system

$$
y_{j}^{\prime}(t)=-p_{j j}(t) y_{j}(t)+\sum_{k=1 ; k \neq j}^{n} p_{j k}(t) y_{k}(t) \quad(j=1, \cdots, n),
$$

in which the coefficients are continuous and satisfy the conditions

$$
p_{k k}(t)-\sum_{j=1 ; j \neq k}^{n}\left|p_{j k}(t)\right| \geqq \delta>0 \quad(k=1, \cdots, n)
$$

tend to zero at the rate $e^{-\delta t}$ as $t \rightarrow+\infty$; in fact

$$
\sum_{j=1}^{n}\left|y_{j}(t)\right| \leqq e^{-\delta t} \sum_{j=1}^{n}\left|y_{j}(0)\right| \quad(t \geqq 0) .
$$

Matrices in which the main diagonal terms predominate in the sense of condition (2) (or a similar condition involving rows rather than columns) have been studied in [1], [2] and [3]. We remark that an application of the estimate (3) in the situation where the coefficients of (1) are time independent allows us indirectly to obtain the bound $\operatorname{Re} \lambda \leqq-\delta$ for the eigenvalues $\lambda$ of a constant matrix with a negative principal diagonal which is dominant in accordance with (2).

Proof. We will first prove the theorem under the additional assumptions that all the coefficients $F_{j k}$ as well as the initial values of the solution are nonnegative. The general result will then be reduced to this situation.

Received by the editors July $26,1971$.

AMS 1970 subject classifications. Primary 34D05; Secondary 15A42.

Key words and phrases. Stability, linear differential system, matrices with dominant main diagonal.

(c) American Mathematical Society 1972 
We begin with the observation that a solution which starts out from nonnegative data will remain nonnegative. This is easily seen if the initial data is strictly positive: $y_{j}(0)>0(j=1, \cdots, n)$. Namely, assume on the contrary, that some of the $y$,'s may become negative. Then there will be a "first" time $t_{0}>0$ that one of the $y_{j}$ 's passes through zero. In the interval $\left[0, t_{0}\right)$ up to this moment, $y_{j}(t)>0(j=1, \cdots, n)$. Hence, since all the coefficients $p_{j k}$ are assumed to be nonnegative, (1) implies that

$$
y_{j}^{\prime}(t)+p_{j j}(t) y_{j}(t) \geqq 0 \quad \text { for } t \in\left[0, t_{0}\right] \text { and } j=1, \cdots, n .
$$

Solving these differential inequalities we find that

$$
y_{j}(t) \geqq y_{j}(0) \exp \left(-\int_{0}^{t} p_{j j}(\tau) d \tau\right) \text { for } t \in\left[0, t_{0}\right] \text { and } j=1, \cdots, n ;
$$

from which it follows that $y_{j}\left(t_{0}\right)>0$ for $j=1, \cdots, n$, contrary to our assumption that one of the $y_{j}$ 's actually becomes zero at $t_{0}$. This establishes our observation when the initial data is strictly positive. In case the initial data, $y_{j}(0)(j=1, \cdots, n)$, is nonnegative, we consider the solutions generated by the perturbed data $y_{j}(0)+\varepsilon(j=1, \cdots, n)$, where $\varepsilon$ is any positive number. By the preceding these solutions will be nonnegative for all $t>0$; passing to the limit as $\varepsilon \rightarrow 0$ we obtain the desired nonnegativity from the continuous dependence of the solutions on the initial data.

If we now add all the equations in the system (1), the application of condition (2) in conjunction with the nonnegativity of the solution yields the estimate

$$
\frac{d}{d t}\left[\sum_{j=1}^{n} y_{j}(t)\right]=-\sum_{k=1}^{n}\left[p_{k k}(t)-\sum_{j=1 ; j \neq k}^{n} p_{j k}(t)\right] y_{k}(t) \leqq-\delta\left[\sum_{k=1}^{n} y_{k}(t)\right] .
$$

Solving this differential inequality for $\sum_{j=1}^{n} y_{j}(t)$ we immediately obtain (3), and this establishes the theorem when the coefficients in (1) and the initial data are all assumed to be nonnegative.

Suppose now that the coefficients in (1) as well as the initial values of the solution are not restricted as to sign. To prove (3) in this case we compare the given solution $y=\left(y_{1}, \cdots, y_{n}\right)$ of (1) with the solution $x=\left(x_{1}, \cdots, x_{n}\right)$ of the system

$$
x_{j}^{\prime}(t)=-p_{j j}(t) x_{j}(t)+\sum_{k=1: k \neq j}^{n}\left|p_{j k}(t)\right| x_{k}(t) \quad(j=1, \cdots, n),
$$

subject to the initial conditions

$$
x_{j}(0)=\left|y_{j}(0)\right| .
$$


We claim that

$$
\left|y_{j}(t)\right| \leqq x_{j}(t) \quad(t \geqq 0) .
$$

This is easily seen by observing that the $x_{j}$ 's satisfy the equivalent system of integral equations

$$
\begin{aligned}
x_{j}(t)= & \left|y_{j}(0)\right| \exp \left(-\int_{0}^{t} p_{j j}(\tau) d \tau\right) \\
& +\int_{0}^{t} \exp \left(-\int_{\tau}^{t} p_{j j}(s) d s\right)\left[\sum_{k=1 ; k \neq j}^{n}\left|p_{j k}(\tau)\right| x_{k}(\tau)\right] d \tau .
\end{aligned}
$$

Whereas, the $\left|y_{j}\right|$ 's satisfy a corresponding system of integral inequalities

$$
\begin{aligned}
\left|y_{j}(t)\right| \leqq & \left|y_{j}(0)\right| \exp \left(-\int_{0}^{t} p_{j j}(\tau) d \tau\right) \\
& +\int_{0}^{t} \exp \left(-\int^{t} p_{j j}(s) d s\right)\left[\sum_{k=1 ; k \neq j}^{n}\left|p_{j k}(\tau)\right|\left|y_{k}(\tau)\right|\right] d \tau .
\end{aligned}
$$

A standard comparison argument then shows that the solution of the equations dominates the solution of the inequalities and (6) follows.

On the other hand, the coefficients of the system (4) are nonnegative and, by virtue of $(5)$, so also are the initial values $x_{j}(0)$. Hence, by what has already been established, (3) applies to the solution $x$ of (4):

$$
\sum_{j=1}^{n} x_{j}(t) \leqq \epsilon^{-\delta t} \sum_{j=1}^{n} x_{j}(0) .
$$

In view of (5) and (6) this implies that

$$
\sum_{j=1}^{n}\left|y_{j}(t)\right| \leqq e^{-\delta t} \sum_{j=1}^{n}\left|y_{j}(0)\right|
$$

which completes the proof of the theorem.

\section{REFERENCES}

1. E. V. Haynsworth, Bounds for determinants with dominant main diagonal, Duke Math. J. 20 (1953), 199-209. MR 14, 837.

2. A. M. Ostrowski, Note on bounds for determinants with dominant principal diagonal, Proc. Amer. Math. Soc. 3 (1952), 26-30. MR 14, 611.

3. G. Baley Price, Bounds for determinants with dominant principal diagonal, Proc. Amer. Miath. Soc. 2 (1951), 497-502. MR 12, 793.

Vanderbilt University, Nashville, Tennessee 37203 